\title{
An $h$-Adaptive RKDG Method for Two-Dimensional Detonation Wave Simulations
}

\author{
Hongqiang $\mathrm{Zhu}^{1}$ and Zhen $\mathrm{Gao}^{2, *}$ \\ ${ }^{1}$ School of Natural Sciences, Nanjing University of Posts and Telecommunications, \\ Nanjing, China. \\ ${ }^{2}$ School of Mathematical Sciences, Ocean University of China, Qingdao, China.
}

Received 10 March 2018; Accepted (in revised version) 1 July 2018.

\begin{abstract}
An $h$-adaptive Runge-Kutta discontinuous Galerkin (RKDG) method with a positivity-preserving technique to simulate classical two-dimensional detonation waves is developed. The KXRCF troubled-cell indicator is used to detect the troubled cells with possible discontinuities or high gradients. At each time-level, an adaptive mesh is generated by refining troubled cells and coarsening others. In order to avoid the situations where detonation front moves too fast and there are not enough cells to describe detonation front before it leaves, a recursive multi-level mesh refinement technique is designed. The numerical results show that for smooth solutions, this $h$-adaptive method does not degrade the optimal convergence order of the nonadaptive method and outperforms it in terms of computational storage for shocked flows.
\end{abstract}

AMS subject classifications: 65M60, 65M99, 35L65

Key words: Runge-Kutta discontinuous Galerkin method, troubled-cell indicator, $h$-adaptive method, detonation wave.

\section{Introduction}

The proper understanding of the detonation waves propagation plays an important role in protecting human lives and avoiding property damages. To study detonation phenomena, various numerical methods have been employed, including second order Godunov scheme [1,27], extended space-time CE/SE method [35], unsplit scheme [23], non-MUSCL-type TVD scheme [31], classical weighted essentially non-oscillatory (WENO) scheme [11,32], optimal WENO-Z scheme [14, 15], hybrid central-WENO scheme [13], Runge-Kutta discontinuous Galerkin (RKDG) method [3,33,34] and adaptive finite volume methods [17] .

It is known that discontinuous Galerkin (DG) finite element method [4,6-8] can handle complicated geometry and $h-p$ adaptation, and can be efficiently implemented on parallel computers. As the result, it finds various applications [5,36,41]. One can relatively easily

*Corresponding author. Email addresses: zhuhq@njupt. edu. cn (H. Zhu), zhengao@ouc.edu.cn (Z. Gao) 
choose an $h$-adaptive strategy to develop a DG method since the mesh refinement or coarsening does not use the continuity through cell interfaces. Moreover, compared to finite volume or finite difference adaptive mesh refinement schemes, DG methods are extremely convenient and computationally efficient in data prolongation and data projection at different mesh levels and in preservation of cell averages for conservation. On the other hand, they are more flexible than continuous finite element methods if there are hanging nodes in adaptive meshes. For more details on $h$-adaptive discontinuous Galerkin methods we refer the reader to $[10,16,26,29,38-40]$.

Let us note that although there is an $h$-adaptive RKDG method for one-dimensional detonation waves [37], all realistic detonation phenomena are essentially three-dimensional. Nevertheless, many important detonation structures can be detected during two-dimensional simulations - e.g. regular or irregular cellular structures and triple-shock Mach intersection in shock interface. Therefore, here we consider an $h$-adaptive RKDG method with troubled-cell indicators for various classical two-dimensional detonation waves. Comparing this method with fixed-mesh RKDG method, we note significant advantage with respect to computational storage and solution quality. In particular, the multi-level mesh refinement technique guaranties that the finest mesh is generated in the neighbourhoods of discontinuities. In addition, the KXRCF troubled-cell indicator is used to detect the discontinuity regions and a high order positivity-preserving technique [33] is adopted to avoid the potential occurrence of negative pressure, density or mass fraction.

This paper is organised as follows. In Section 2, we introduce the RKDG method. An $h$-adaptive RKDG method for the two-dimensional reactive Euler equations and its implementation are considered in Section 3. The performance of this adaptive technique is demonstrated on several classical examples in Section 4 and our concluding remarks are in Section 5.

\section{Review}

We consider the system of two-dimensional conservation laws with a source term

$$
\mathbf{w}_{t}+\mathbf{f}(\mathbf{w})_{x}+\mathbf{g}(\mathbf{w})_{y}=\mathbf{s}(\mathbf{w}), \quad t>0, \quad(x, y) \in \Omega,
$$

where $\mathbf{w}$ is the conserved variable vector, $\mathbf{s}(\mathbf{w})$ the source vector and $\mathbf{f}(\mathbf{w})$ and $\mathbf{g}(\mathbf{w})$ are the flux vectors. The two-dimensional governing equations for modeling the ideal gaseous detonation has the form

$$
\left(\begin{array}{c}
\rho \\
\rho u \\
\rho v \\
E \\
\rho f_{1}
\end{array}\right)_{t}+\left(\begin{array}{c}
\rho u \\
\rho u^{2}+P \\
\rho u v \\
(E+p) u \\
\rho f_{1} u
\end{array}\right)_{x}+\left(\begin{array}{c}
\rho v \\
\rho u v \\
\rho v^{2}+P \\
(E+p) v \\
\rho f_{1} v
\end{array}\right)_{y}=\left(\begin{array}{c}
0 \\
0 \\
0 \\
0 \\
\omega
\end{array}\right)
$$

where $\rho$ is the density, $(u, v)$ the velocity vector, $P$ the pressure, and $f_{1} \in[0,1]$ the reactant mass fraction. Let us also note that the total energy $E$ of the system can be expressed in 
the form

$$
E=\frac{P}{\gamma-1}+\frac{1}{2} \rho\left(u^{2}+v^{2}\right)+\rho f_{1} q_{0}
$$

where $\rho f_{1} q_{0}$ is the energy, generated through a chemical reaction, $q_{0}$ the heat-release parameter and $\gamma$ the ratio of specific heats. We denote by $K(T)$ the reaction rate. It can be written in the Arrhenius form

$$
K(T)=K e^{-E_{a} / T},
$$

where $T=P /(\rho R)$ is the temperature, $R$ a suitably normalised specific gas constant - e.g. throughout this study $R=1, E_{a}$ the activation-energy parameter, and $K$ a pre-exponential factor (stiffness coefficient) defining spatial and temporal scales. The reaction rate $K(T)$ could be also expressed in the Heaviside form - viz.

$$
K(T)= \begin{cases}1 / \sigma, & T \geq E_{a} \\ 0, & T<E_{a}\end{cases}
$$

with the reaction time $\sigma$ such that $1 / \sigma \approx K$. Having defined the reaction time $K$, we consider the source term

$$
\omega=-K(T) \rho f_{1},
$$

for the rate of species production due to chemical reaction.

Let us now introduce an RKDG method for the model problem (2.1). Using a rectangular mesh $\mathscr{M}_{h}$ on the domain $\Omega$, we seek an approximate solution $\mathbf{w}_{h}(x, y, t)$ in the finite element space of discontinuous piecewise polynomials

$$
\mathbf{V}_{h}^{k}=\left\{\mathbf{v} \in\left[L^{2}(\Omega)\right]^{5}:\left.\mathbf{v}\right|_{K} \in\left[\mathbb{P}^{k}(K)\right]^{5} \quad \text { for any } \quad K \in \mathscr{M}_{h}\right\},
$$

where $\mathbb{P}^{k}(K)$ is the set of polynomials of total degree at most $k$ on the element $K$. It is known that the dimension of the space $\mathbf{V}_{h}^{k}$ is $Q_{k}+1$ with $Q_{k}=k(k+3) / 2$. On the reference cell $[-1 / 2,1 / 2] \times[-1 / 2,1 / 2]$, we consider the following orthogonal basis for $\mathbf{V}_{h}^{k}$ :

$$
\begin{array}{ll}
k=1: & \phi_{0}=1, \quad \phi_{1}=x, \quad \phi_{2}=y, \\
k=2: & \phi_{0}, \phi_{1}, \phi_{2}, \phi_{3}=x^{2}-1 / 12, \quad \phi_{4}=x y, \quad \phi_{5}=y^{2}-1 / 12 .
\end{array}
$$

Let $\left(x_{K}, y_{K}\right)$ be the center of the rectangle $K$ and $\Delta x_{K}$ and $\Delta x_{K}$ be the side lengths of $K$ in the directions $x$ and $y$, respectively. Then the local orthogonal basis over cell $K$ is given by

$$
\phi_{l}^{(K)}(x, y):=\phi_{l}\left(\frac{x-x_{K}}{\Delta x_{K}}, \frac{y-y_{K}}{\Delta y_{K}}\right), \quad l=0, \cdots, Q_{k} .
$$

The numerical solution $\mathbf{w}_{h}(x, y, t) \in \mathbf{V}_{h}^{k}$ of the problem (2.1) is sought in the form

$$
\left.\mathbf{w}_{h}(x, y, t)\right|_{K}=\sum_{l=0}^{Q_{k}} \mathbf{w}_{K}^{(l)}(t) \phi_{l}^{(K)}(x, y),
$$


where $\mathbf{w}_{K}^{(l)}(t), l=0, \cdots, Q_{k}$ are the degrees of freedom. In particular, $\mathbf{w}_{K}^{(0)}(t)$ is the vector of cell averages of $\mathbf{w}_{h}$ over $K$.

We now can describe the RKDG scheme. Replacing $\mathbf{w}$ by $\mathbf{w}_{h}$ in the Eq. (2.1), we successively multiply the results by the local bases of every element and integrate the corresponding products over the computational cells. The integration by parts leads to the following weak formulation of the problem (2.1):

Find a $\mathbf{w}_{h}$ in $\mathbf{V}_{h}^{k}$ such that for all test functions $\phi_{l}^{(K)}$ with $l=0, \cdots, Q_{k}$ and all $K \in \mathscr{M}_{h}$ the relations

$$
\begin{aligned}
& \frac{d}{d t} \int_{K} \mathbf{w}_{h} \phi_{l}^{(K)} d x d y-\int_{K} \mathbf{F}\left(\mathbf{w}_{h}\right) \cdot \nabla \phi_{l}^{(K)} d x d y \\
& \quad+\sum_{e \in \partial K} \int_{e} \mathbf{F}\left(\mathbf{w}_{h}\right) \cdot n_{e, K} \phi_{l}^{(K)} d s=\int_{K} \mathbf{s}\left(\mathbf{w}_{h}\right) \phi_{l}^{(K)} d x d y
\end{aligned}
$$

where $\mathbf{F}\left(\mathbf{w}_{h}\right)=\left(\mathbf{f}\left(\mathbf{w}_{h}\right), \mathbf{g}\left(\mathbf{w}_{h}\right)\right)$ and $n_{e, K}$ is the outward unit normal to the edge $e$, hold.

We note that for $h$-adaptive meshes method with hanging nodes, it is important that the intervals $e \subset \partial K$ in the Eq. (2.6) are elementary edges without sub-edges. It guaranties the mass conservation.

The volume integrals $\int_{K} \mathbf{F}\left(\mathbf{w}_{h}\right) \cdot \nabla \phi_{l}^{(K)} d x d y$ and $\int_{K} \mathbf{s}\left(\mathbf{w}_{h}\right) \phi_{l}^{(K)} d x d y$ can be computed by numerical quadratures, providing a sufficient accuracy level. The line integral in the Eq. (2.6) is usually discretised by the Gaussian quadratures

$$
\int_{e} \mathbf{F}\left(\mathbf{w}_{h}\right) \cdot n_{e, K} \phi_{l}^{(K)} d s \approx|e| \sum_{l=1}^{m} \omega_{l} \mathbf{F}\left(\mathbf{w}_{h}\left(G_{l}, t\right)\right) \cdot n_{e, K} \phi_{l}^{(K)}\left(G_{l}, t\right)
$$

with $\mathbf{F}\left(\mathbf{w}_{h}\left(G_{l}, t\right)\right) \cdot n_{e, K}$ replaced by a monotone numerical flux. In this work, we use a simple Lax-Friedrichs flux — viz.

$$
\begin{aligned}
\mathbf{F}\left(\mathbf{w}_{h}\left(G_{l}, t\right)\right) \cdot n_{e, K} \approx & \frac{1}{2}\left[\left(\mathbf{F}\left(\mathbf{w}_{h}^{-}\left(G_{l}, t\right)\right)+\mathbf{F}\left(\mathbf{w}_{h}^{+}\left(G_{l}, t\right)\right)\right) \cdot n_{e, K}\right. \\
& \left.-\alpha\left(\mathbf{w}_{h}^{+}\left(G_{l}, t\right)-\mathbf{w}_{h}^{-}\left(G_{l}, t\right)\right)\right],
\end{aligned}
$$

where $\alpha$ is an upper bound for the eigenvalues of the Jacobian $\left(\partial \mathbf{F}\left(\mathbf{w}_{h}\right) / \partial \mathbf{w}\right) \cdot n_{e, K}$, and $\mathbf{w}_{h}^{-}$and $\mathbf{w}_{h}^{+}$are the traces of $\mathbf{w}_{h}$ at the Gaussian point $G_{l}$ inside and outside the cell $K$ respectively.

Using (2.5), we write the first term in (2.6) in the form $c_{l} \Delta x_{K} \Delta y_{K}\left(d \mathbf{w}_{K}^{(l)}(t) / d t\right)$ with the constants

$$
c_{l}=\int_{-1 / 2}^{1 / 2} \int_{-1 / 2}^{1 / 2} \phi_{l}^{2}(x, y) d x d y, \quad l=0, \cdots, Q_{k} .
$$

Thus the semi-discrete scheme (2.6-2.7) is a system of ordinary differential equations for $\mathbf{w}_{K}^{(l)}(t), l=0, \cdots, Q_{k}, K \in \mathscr{M}_{h}$. This system, combined with a suitable time discretisation - e.g. with total variation diminishing RK method [28], forms an RKDG scheme. Here, 
we use the following third order RK time stepping

$$
\begin{aligned}
& \Phi^{(1)}=\Phi^{n}+\Delta t \mathscr{L}\left(\Phi^{n}\right), \\
& \Phi^{(2)}=\frac{3}{4} \Phi^{n}+\frac{1}{4} \Phi^{(1)}+\frac{1}{4} \Delta t \mathscr{L}\left(\Phi^{(1)}\right), \\
& \Phi^{n+1}=\frac{1}{3} \Phi^{n}+\frac{2}{3} \Phi^{(2)}+\frac{2}{3} \Delta t \mathscr{L}\left(\Phi^{(2)}\right)
\end{aligned}
$$

for the ODE system $\Phi_{t}=\mathscr{L}(\Phi)$

\section{Algorithm and Implementation Details}

In this section, we develop and employ an $h$-adaptive RKDG scheme to two-dimensional detonation wave problems.

\subsection{Grid and data structure}

For $h$-adaptive rectangular meshes, there are two types of data structures - viz. blockbased and cell-based, each with own advantages. Here, we prefer to use the cell-based structures because of their high flexibility in refinement and coarsening. More exactly, each cell in the initial partition of the computational domain is considered as the root of a tree. Mesh refinement is achieved by dividing a cell into four new cells - children, of equal size. These four new cells simultaneously generated by a single division, are called GROUP and the only cells from the same GROUP can be used in mesh coarsening. Fig. 1 shows the sketches of both operations.

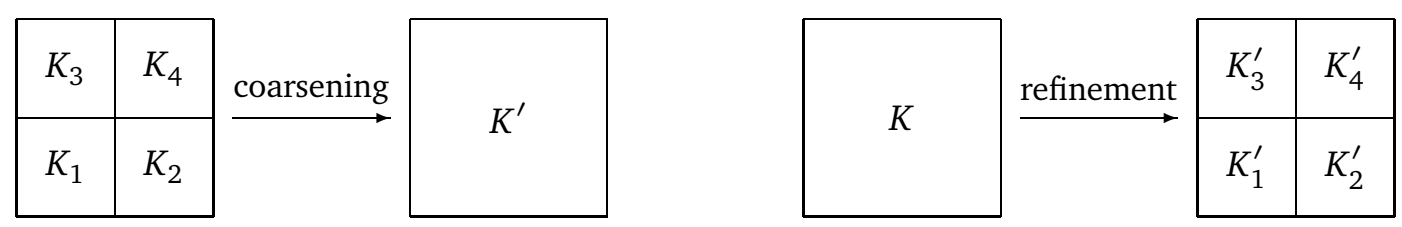

Figure 1: Local mesh transformation. Left: coarsening. Right: refinement.

A cell is called a leaf cell if it does not have any children. All the leaf cells constitute a computational mesh. Each leaf cell has a variable $L$ denoting its mesh-level, defined by the number of divisions needed to obtain this cell. For roots, the parameter $L$ is set to zero and it grows by 1 after a division and diminishes by 1 after a merger. We also need a maximal mesh-level, denoted by $L E V$, to restrict the fineness of the mesh. Finally, we describe the data structure associated with each leaf cell and non-leaf cell. For non-leaf cells, the associated data contain the cell coordinates, a mesh-level and the pointers to the parent and children in the tree. For leaf cells, there are additional data stored - e.g. the degree of freedom for solutions, pointers for neighboring cells, indicators for mesh refinement and coarsening, and indicator for boundary cells [39]. 


\subsection{Limiter and solution reconstruction method}

The solutions of detonation waves contain discontinuities and sharp gradients. Therefore, a nonlinear limiter in the RKDG method to detect discontinuities and control the corresponding spurious oscillations during the solution reconstruction is needed. We note that the troubled-cell indicators for the detection of discontinuous regions, also control the mesh adaptation.

The complexity of two-dimensional $h$-adaptive meshes with hanging nodes causes problems with the solution reconstruction part of the limiter. Thus, the cells can have different number of neighbors, which can be of different size. Moreover, the cell can have different neighbors at different time levels. The mesh varies not only in time but also in space. In this work, we use the approach [39]. If a cell $K$ requires a solution reconstruction, we build a local uniform mesh of $(2 k+1) \times(2 k+1)$ cells such that $K$ is located in the center of the mesh constructed - cf. Fig. 2.

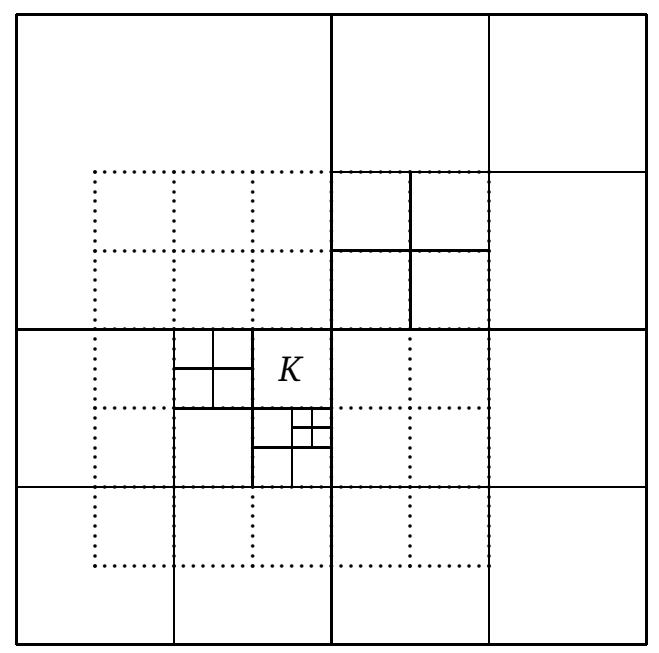

Figure 2: Dotted lines: Imaginary local uniform mesh of $K, k=2$.

These imaginary cells are used in the reconstruction of the solution in $K$ instead of the real ones. Since the imaginary local mesh is uniformly rectangular, the WENO solution reconstruction [25] can be applied directly. The only problem left is to derive the cell averages for the imaginary local mesh. But they can be easily found from the $L^{2}$-projection formula (3.1).

Since the initial mesh and the imaginary local one are uniformly rectangular, a cell $\tilde{K}$ in the imaginary local mesh either coincides with a real cell $K$, or is a subcell of a real cell, or a union of several real cells $K_{1}, \cdots, K_{m}$. In the first case, the cell averages of $\tilde{K}$ are equal to the cell averages of $K$. In the second, the cell averages of $\tilde{K}$ can be found from the Eq. (3.1). In the remaining case, we can use the Eq. (3.1) to obtain $\mathbf{w}_{\tilde{K}}^{(0)}=\left(\sum_{i=1}^{m} \mathbf{w}_{K_{i}}^{(0)} S_{K_{i}}\right) / S_{\tilde{K}}$, where $S_{K_{i}}$ and $S_{\tilde{K}}$ are, respectively, the areas of $K_{i}$ and $\tilde{K}$. 


\subsection{Troubled-cell indicator}

Many troubled-cell indicators, including widely-used in TVB scheme [8], involve cell averages of $\mathbf{w}_{h}$ over neighboring cells. However, they cannot be directly applied to $h$-adaptive meshes. Instead, one can use the shock-detection technique based indicator KXRCF, introduced by Krivodonova et al. [20]. As numerical tests in [24,38] show, it is usually more efficient and reliable.

The KXRCF troubled-cell indicator works as follows. Let $\partial K^{-}$and $\partial K^{+}$be the parts of the cell $K$ boundary, where the flow is directed in or out of $K$, respectively. We say that $K$ is a troubled cell, if

$$
\frac{\left|\int_{\partial K^{-}}\left(\left.\eta^{h}\right|_{K}-\left.\eta^{h}\right|_{K_{n}}\right) d s\right|}{\left.h_{K}^{(k+1) / 2}\left|\partial K^{-}\right||| \eta^{h}\right|_{K} \|}>1,
$$

where $h_{K}$ is the radius of the circumscribed circle for $K, K_{n}$ the neighbor of $K$ at $\partial K^{-}$and $\|\cdot\|$ is the maximum norm related to the quadrature points. The term $\eta$ can mean the density, pressure, energy or their projected variables in local characteristic field. Moreover, the combinations of these variables can also be used to identify a troubled cell. Here, $\eta$ is the density and this lead to satisfactory numerical results.

\subsection{Algorithm}

In $h$-adaptive methods, it is important to identify where and how the corresponding mesh should be refined or coarsened. In particular, for the detonation waves simulating here, the meshes should be refined near the discontinuities in order to catch their sharp transitions. Therefore, following the ideas of [37], we coarse the mesh over untroubled cells and refine it for troubled cells. In order to avoid the situations where detonation front moves too fast and there are not enough cells to describe detonation front before it leaves, mesh refinement is performed in a recursive way. More specifically, the target cell $K$ is refined if it satisfies the conditions:

(R1) $K$ is a troubled cell.

(R2) The mesh-level $L$ on $K$ is lower than the maximum mesh-level $L E V$.

In this case, each of the newly generated four cells is further refined. The procedure is recursively applied to every new cell, provided it satisfies the conditions above. Thus, the finest mesh can be generated within one time step whenever needed.

Unfortunately, at the moment, there are no errorless troubled-cell indicators and if a cell is falsely identified as a troubled cell, an extra mesh refinement occurs. On the other hand, if a cell is falsely identified as an untroubled cell, it may be falsely coarsened. The former is acceptable while the latter is not. Extra refinements can simply increase the computational cost, but a false merger often diminishes the resolution of numerical solutions. Let $N F L$ denote the number of merger-forbidden time-levels after a division - i.e. a GROUP of cells cannot be merged in the following NFL time-levels from the time-level they are generated. To achieve this, for each computational cell we need a new variable $A I \in[0, N F L+1]$, 
called adaptive indicator, to record the difference between the current time-level and the time-level where it was generated by a division. At each time-level, the $A I$ is initially set to $\min (A I+1, N F L+1)$. For every new cell, arising in a division we let $A I=0$, and we set $A I=N F L$ for the new merged cells. We now can provide coarsening criteria - viz. four cells are merged if:

(C1) They form a GROUP.

(C2) They all are untroubled.

(C3) $A I=N F L+1$ for all of them.

Let us now describe the full $h$-adaptive algorithm in more details.

Algorithm 3.1 (h-adaptive RKDG scheme)

Given a maximum mesh-level $L E V$, the number of merger-forbidden time-levels $N F L$ :

- The initial set up. The algorithm starts from a uniform rectangular mesh $\mathscr{M}_{h}^{0}$ as the root grid. Consider the $L^{2}$-projection of initial data $\mathbf{w}(x, y, t=0)$ on $\mathscr{M}_{h}^{0}$. For every root cell $K \in \mathscr{M}_{h}^{0}$ we have

$$
\left\{\mathbf{w}_{K}^{(l), 0}: l=0, \cdots, Q_{k}\right\}, \quad\left\{L_{K}^{0}\right\}=0, \quad\left\{A I_{K}^{0}\right\}=N F L+1 .
$$

- Solution evolution from $t^{n}$ to $t^{n+1}$, for $n=0,1, \cdots$.

1. For all leaf cells, set $A I_{K}^{n}=\min \left(A I_{K}^{n}+1, N F L+1\right)$.

2. Mesh refinement and coarsening: Each cell in the current mesh is marked to be refined, coarsened, or kept unchanged via troubled-cell indicator.

- Each cell is recursively quartered, stopping when conditions (R1)-(R2) fail. At every quartering, there are four newly generated cells (children), with their mesh-level increased by one from the parent cell and $A I=0$. On the new cells the corresponding polynomial is obtained by the data prolongation mechanism from Section 3.5.

- If four cells satisfy conditions (C1)-(C3) they are removed, the meshlevel $L$ is decreased by one and $A I$ is set to NFL for the new leaf cell. The corresponding polynomial on it is obtained by the data projection discussed in Section 3.5.

3. Evolve solution: Evolve the solution on the current mesh from $t_{n}$ to $t_{n+1}$ to update $\left\{\mathbf{w}^{(l), n+1}: l=0, \cdots, Q_{k}, \forall K \in \mathscr{M}_{h}^{n+1}\right\}$ by applying the RKDG procedure. 
Remark 3.1. At the initial time, the projection of initial conditions on a new adaptive mesh is used instead of the approximate solution on the initial mesh. This helps to resolve the features of initial conditions.

Compared to the standard RKDG scheme, the proposed $h$-adaptive technique adds a mesh-adaptation step immediately after the solution at each time-level is obtained. The limiter is applied after each RK inner stage except the case when it is applied to the projected solution after the mesh adaptation when the whole RK time step is completed. Adaptive time stepping treatment $[9,19,22]$ can improve the time discretisation efficiency, but it is not considered here. In the RK method, global time steps proportional to the smallest cell size at each time step are used. To avoid the potential appearance of negative density, negative pressure or mass fraction and to make the algorithm more robust, the high order positivity-preserving technique [33] for reactive Euler equations is applied immediately after the limiter.

Let $\mathbf{w}_{K}=\left(\rho_{K},(\rho u)_{K},(\rho v)_{K}, E_{K},\left(\rho f_{1}\right)_{K}\right)^{T}$ and $\overline{\mathbf{w}}_{K}=\left(\bar{\rho}_{K}, \overline{\rho u}_{K}, \overline{\rho \nu}_{K}, \bar{E}_{K}, \overline{\rho f_{1 K}}\right)^{T}$ denote respectively the DG polynomial and the cell average of $\mathbf{w}$ on a cell $K$. Choose a small number $\varepsilon\left(10^{-13}\right.$ in the computation) such that $\bar{\rho}_{K} \geq \varepsilon$ for all $K$. Let $S_{K}$ be the set of all numerical quadrature points on $K$ in the DG scheme. For each computational cell $K \in \mathscr{M}_{h}$, the density positivity is enforced by

$$
\widehat{\rho}_{K}(x, y)=\theta_{K}^{1}\left[\rho_{K}(x, y)-\bar{\rho}_{K}\right]+\bar{\rho}_{K}, \quad \theta_{K}^{1}=\min _{(x, y) \in S_{K}}\left\{1,\left|\frac{\bar{\rho}_{K}-\varepsilon}{\bar{\rho}_{K}-\rho_{K}(x, y)}\right|\right\} .
$$

Similarly, we enforce the reactant mass fraction by

$$
{\widehat{\rho f_{1 K}}}_{1}(x, y)=\theta_{K}^{2}\left[\left(\rho f_{1}\right)_{K}(x, y)-\overline{\rho f_{1 K}}\right]+\overline{\rho f_{1 K}}, \quad \theta_{K}^{2}=\min _{(x, y) \in S_{K}}\left\{1,\left|\frac{\overline{\rho f_{1 K}}}{\overline{\rho f_{1 K}}-\left(\rho f_{1}\right)_{K}(x, y)}\right|\right\} \text {, }
$$

and the pressure positivity by

$$
\widetilde{\mathbf{w}}_{K}(x, y)=\theta_{K}\left[\widehat{\mathbf{w}}_{K}(x, y)-\overline{\mathbf{w}}_{K}\right]+\overline{\mathbf{w}}_{K}, \quad \theta_{K}=\min _{(x, y) \in S_{K}}\left\{1,\left|\frac{p\left(\overline{\mathbf{w}}_{K}\right)}{p\left(\overline{\mathbf{w}}_{K}\right)-p\left(\widehat{\mathbf{w}}_{K}(x, y)\right)}\right|\right\},
$$

where $\widehat{\mathbf{w}}_{K}=\left(\widehat{\rho}_{K},(\rho u)_{K},(\rho v)_{K}, E_{K}, \widehat{\rho f}_{1 K}\right)^{T}$.

\subsection{Data prolongation and data projection}

An $L^{2}$-projection is used for data prolongation between the different mesh levels. Suppose we already know $\mathbf{w}_{h}$ on the mesh $\mathscr{M}_{h}$, and have to determine its $L^{2}$-projection on a new cell $K^{\prime}$. This will be a new polynomial function $\mathbf{w}_{h}^{\prime}$, which satisfies the equations

$$
\int_{K^{\prime}} \mathbf{w}_{h}^{\prime} \phi_{l}^{\left(K^{\prime}\right)}(x, y) d x d y=\int_{K^{\prime}} \mathbf{w}_{h} \phi_{l}^{\left(K^{\prime}\right)}(x, y) d x d y, \quad l=0, \cdots, Q_{k} .
$$


Similar to the Eq. (2.5), we denote by $\mathbf{w}_{K^{\prime}}^{(l)}, l=0, \cdots, Q_{k}$ the degree of freedom associated with $\mathbf{w}_{h}^{\prime}$ on the cell $K^{\prime}$, so that

$$
\mathbf{w}_{K^{\prime}}^{(l)}=\frac{1}{c_{l} \Delta x_{K^{\prime}} \Delta y_{K^{\prime}}} \int_{K^{\prime}} \mathbf{w}_{h} \phi_{l}^{\left(K^{\prime}\right)}(x, y) d x d y .
$$

For $l=0$, this formula implies the conservation. The integral in Eq. (3.1) can be computed exactly, since $\mathbf{w}_{h}$ is a piecewise polynomial. Now we can provide the formulas of data projection and data prolongation between fine and coarse meshes.

- Data projection: If four cells $K_{1}, K_{2}, K_{3}, K_{4}$ are merged to a new cell $K^{\prime}-$ cf. the left graph in Fig. 1, then for the DG scheme with $P_{2}$ polynomial space, the new degrees of freedom obtained from (3.1) are

$$
\begin{aligned}
\mathbf{w}_{K^{\prime}}^{(0)}= & \frac{1}{4}\left(\mathbf{w}_{K_{1}}^{(0)}+\mathbf{w}_{K_{2}}^{(0)}+\mathbf{w}_{K_{3}}^{(0)}+\mathbf{w}_{K_{4}}^{(0)}\right), \\
\mathbf{w}_{K^{\prime}}^{(1)}= & \frac{3}{4}\left(-\mathbf{w}_{K_{1}}^{(0)}+\mathbf{w}_{K_{2}}^{(0)}-\mathbf{w}_{K_{3}}^{(0)}+\mathbf{w}_{K_{4}}^{(0)}\right)+\frac{1}{8}\left(\mathbf{w}_{K_{1}}^{(1)}+\mathbf{w}_{K_{2}}^{(1)}+\mathbf{w}_{K_{3}}^{(1)}+\mathbf{w}_{K_{4}}^{(1)}\right), \\
\mathbf{w}_{K^{\prime}}^{(2)}= & \frac{3}{4}\left(-\mathbf{w}_{K_{1}}^{(0)}-\mathbf{w}_{K_{2}}^{(0)}+\mathbf{w}_{K_{3}}^{(0)}+\mathbf{w}_{K_{4}}^{(0)}\right)+\frac{1}{8}\left(\mathbf{w}_{K_{1}}^{(2)}+\mathbf{w}_{K_{2}}^{(2)}+\mathbf{w}_{K_{3}}^{(2)}+\mathbf{w}_{K_{4}}^{(2)}\right), \\
\mathbf{w}_{K^{\prime}}^{(3)}= & \frac{15}{16}\left(-\mathbf{w}_{K_{1}}^{(1)}+\mathbf{w}_{K_{2}}^{(1)}-\mathbf{w}_{K_{3}}^{(1)}+\mathbf{w}_{K_{4}}^{(1)}\right)+\frac{1}{16}\left(\mathbf{w}_{K_{1}}^{(3)}+\mathbf{w}_{K_{2}}^{(3)}+\mathbf{w}_{K_{3}}^{(3)}+\mathbf{w}_{K_{4}}^{(3)}\right), \\
\mathbf{w}_{K^{\prime}}^{(4)}= & \frac{9}{4}\left(\mathbf{w}_{K_{1}}^{(0)}-\mathbf{w}_{K_{2}}^{(0)}-\mathbf{w}_{K_{3}}^{(0)}+\mathbf{w}_{K_{4}}^{(0)}\right)+\frac{3}{8}\left(-\mathbf{w}_{K_{1}}^{(1)}-\mathbf{w}_{K_{2}}^{(1)}+\mathbf{w}_{K_{3}}^{(1)}+\mathbf{w}_{K_{4}}^{(1)}\right) \\
& +\frac{3}{8}\left(-\mathbf{w}_{K_{1}}^{(2)}+\mathbf{w}_{K_{2}}^{(2)}-\mathbf{w}_{K_{3}}^{(2)}+\mathbf{w}_{K_{4}}^{(2)}\right)+\frac{1}{16}\left(\mathbf{w}_{K_{1}}^{(4)}+\mathbf{w}_{K_{2}}^{(4)}+\mathbf{w}_{K_{3}}^{(4)}+\mathbf{w}_{K_{4}}^{(4)}\right), \\
\mathbf{w}_{K^{\prime}}^{(5)}= & \frac{15}{16}\left(-\mathbf{w}_{K_{1}}^{(2)}-\mathbf{w}_{K_{2}}^{(2)}+\mathbf{w}_{K_{3}}^{(2)}+\mathbf{w}_{K_{4}}^{(2)}\right)+\frac{1}{16}\left(\mathbf{w}_{K_{1}}^{(5)}+\mathbf{w}_{K_{2}}^{(5)}+\mathbf{w}_{K_{3}}^{(5)}+\mathbf{w}_{K_{4}}^{(5)}\right) .
\end{aligned}
$$

For the DG scheme with $P_{1}$ polynomial space, only the first three formulas are needed.

- Data prolongation: If a cell $K$ is divided equally into four subcells $K_{1}^{\prime}, K_{2}^{\prime}, K_{3}^{\prime}, K_{4}^{\prime}-$ cf. the right graph in Fig. 1 , the new degrees of freedom for $k=2$ and $l=1,2,3,4$ can be computed as follows:

$$
\begin{aligned}
\mathbf{w}_{K_{l}^{\prime}}^{(0)} & =\mathbf{w}_{K}^{(0)}+\lambda_{x}^{(l)} \mathbf{w}_{K}^{(1)}+\lambda_{y}^{(l)} \mathbf{w}_{K}^{(2)}+\lambda_{x}^{(l)} \lambda_{y}^{(l)} \mathbf{w}_{K}^{(4)}, \\
\mathbf{w}_{K_{l}^{\prime}}^{(1)} & =\frac{1}{2} \mathbf{w}_{K}^{(1)}+\lambda_{x}^{(l)} \mathbf{w}_{K}^{(3)}+\frac{1}{2} \lambda_{y}^{(l)} \mathbf{w}_{K}^{(4)}, \\
\mathbf{w}_{K_{l}^{\prime}}^{(2)} & =\frac{1}{2} \mathbf{w}_{K}^{(2)}+\frac{1}{2} \lambda_{x}^{(l)} \mathbf{w}_{K}^{(4)}+\lambda_{y}^{(l)} \mathbf{w}_{K}^{(5)}, \\
\mathbf{w}_{K_{l}^{\prime}}^{(3)} & =\frac{1}{4} \mathbf{w}_{K}^{(3)}, \quad \mathbf{w}_{K_{l}^{\prime}}^{(4)}=\frac{1}{4} \mathbf{w}_{K}^{(4)}, \quad \mathbf{w}_{K_{l}^{\prime}}^{(5)}=\frac{1}{4} \mathbf{w}_{K}^{(5)},
\end{aligned}
$$

where $\lambda_{x}^{(l)}=(-1)^{l} / 4, l=1, \cdots, 4$ and $\lambda_{y}^{(1)}=\lambda_{y}^{(2)}=-1 / 4, \lambda_{y}^{(3)}=\lambda_{y}^{(4)}=1 / 4$. For $k=1$, one can use the same formulas with $\mathbf{w}_{K}^{(3)}=\mathbf{w}_{K}^{(4)}=\mathbf{w}_{K}^{(5)}=0$. 


\section{Numerical Results}

In this section, the performance of the proposed two-dimensional $h$-adaptive RKDG method is illustrated by the series of benchmark examples and the comparison with the fixed-mesh RKDG method. For convenience, fixed-mesh RKDG method and $h$-adaptive RKDG method are called nonadaptive and adaptive methods, respectively. Moreover, we use $N F L=2$ and $L E V=4$. Other sets of these parameters are also tested. The corresponding results are not presented since the parameters chosen already demonstrate the power of the adaptive method.

Example 4.1 (Smooth solutions. Convergence - cf. Toro \& Titarev [30]). We first test the grid convergence of the method in the case of smooth solutions. Consider the twodimensional inviscid Burgers' equation with a time-dependent source term

$$
\begin{aligned}
& w_{t}+\left(\frac{1}{2} w^{2}\right)_{x}+\left(\frac{1}{2} w^{2}\right)_{y}=s(x, y, t, w), \\
& s(x, y, t, w)=\pi(w-1)[\cos (\pi(x-t)) \sin (\pi(y-t))+\sin (\pi(x-t)) \cos (\pi(y-t))],
\end{aligned}
$$

the initial conditions

$$
w(x, y, 0)=w_{0}(x, y)=\sin (\pi x) \sin (\pi y), \quad x, y \in[-1,1],
$$

and periodic boundary conditions. The exact solution of this problem is $w(x, y, t)=w_{0}(x-$ $t, y-t)$.

Since solution is continuous, it is highly likely that KXRCF troubled-cell indicator will discover no troubled cell, so that no mesh adaptation will be needed. Therefore, we carry out random mesh refinement and coarsening without the limiter. Every computational cell is randomly identified as a troubled cell with the probability $p$. Besides, the random mesh adaptation is only implemented in the region $[-0.5,0.5] \times[-0.5,0.5]$. This does not influence the effectiveness of the test but help to treat the boundary conditions.

For $t=1.0$, the initial meshes $N_{0} \times M_{0}=8 \times 8,16 \times 16, \cdots, 128 \times 128$ and probability $p=0.1$ the errors and convergence orders are listed in Table 1 . We note $(k+1)$-th order of accuracy for $P_{1}-$ i.e. for $k=1$ and for $P_{2}-$ i.e. for $k=2$. This shows that the adaptive method proposed does not reduce the optimal order of accuracy of the nonadaptive method. In addition, Fig. 3 demonstrates an adaptive mesh generated by random mesh adaptation with an $8 \times 8$ initial mesh in $P_{2}$-case.

Example 4.2 (Discontinuous solutions. Convergence - cf. Wang et al. [33]). Here, we test the grid convergence of the adaptive method in the case of discontinuous solutions. The Eq. (2.2) is considered on the domain $[0,2] \times[0,2]$ along with the initial conditions

$$
\left(\rho, u, v, p, f_{1}\right)= \begin{cases}(1,0,0,80,0), & x^{2}+y^{2} \leq 0.36 \\ \left(1,0,0,10^{-9}, 1\right), & \text { otherwise }\end{cases}
$$

with reflective boundary conditions on the bottom and the left boundary and with the final time $t=0.2$. In the adaptive method, we start with the uniform rectangular mesh 
Table 1: Example 4.1. Errors and convergence orders of numerical solution, $t=1.0$.

\begin{tabular}{||rr|rc|cc|cc||}
\hline & $N_{0} \times M_{0}$ & $L^{1}$ error & order & $L^{2}$ error & order & $L^{\infty}$ error & order \\
\hline$P_{1}$ & $8 \times 8$ & $1.89 \mathrm{E}-01$ & & $1.21 \mathrm{E}-01$ & & $2.51 \mathrm{E}-01$ & \\
& $16 \times 16$ & $3.62 \mathrm{E}-02$ & 2.36 & $2.41 \mathrm{E}-02$ & 2.31 & $5.25 \mathrm{E}-02$ & 2.24 \\
& $32 \times 32$ & $6.90 \mathrm{E}-03$ & 2.38 & $4.95 \mathrm{E}-03$ & 2.27 & $1.28 \mathrm{E}-02$ & 2.02 \\
& $64 \times 64$ & $1.45 \mathrm{E}-03$ & 2.24 & $1.13 \mathrm{E}-03$ & 2.12 & $3.36 \mathrm{E}-03$ & 1.92 \\
& $128 \times 128$ & $3.34 \mathrm{E}-04$ & 2.12 & $2.75 \mathrm{E}-04$ & 2.04 & $8.56 \mathrm{E}-04$ & 1.97 \\
\hline$P_{2}$ & $8 \times 8$ & $1.54 \mathrm{E}-02$ & & $1.00 \mathrm{E}-02$ & & $4.15 \mathrm{E}-02$ & \\
& $16 \times 16$ & $1.76 \mathrm{E}-03$ & 3.12 & $1.19 \mathrm{E}-03$ & 3.07 & $5.30 \mathrm{E}-03$ & 2.96 \\
& $32 \times 32$ & $2.14 \mathrm{E}-04$ & 3.02 & $1.53 \mathrm{E}-04$ & 2.93 & $7.73 \mathrm{E}-04$ & 2.75 \\
& $64 \times 64$ & $2.68 \mathrm{E}-05$ & 2.99 & $2.01 \mathrm{E}-05$ & 2.92 & $1.01 \mathrm{E}-04$ & 2.93 \\
& $128 \times 128$ & $3.41 \mathrm{E}-06$ & 2.97 & $2.64 \mathrm{E}-06$ & 2.93 & $1.38 \mathrm{E}-05$ & 2.87 \\
\hline
\end{tabular}
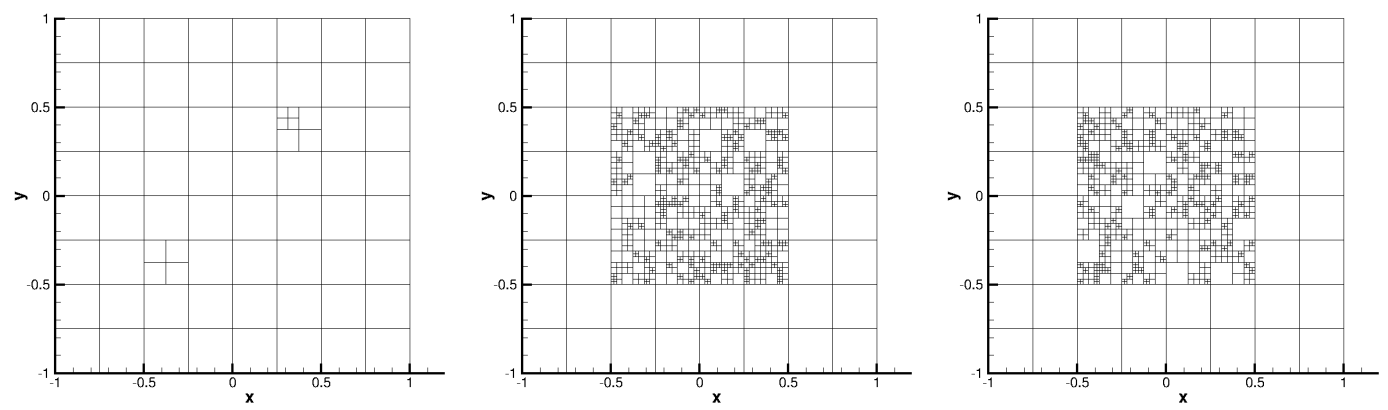

Figure 3: Example 4.1. Adaptive mesh history, $P_{2}$-case with $8 \times 8$ initial mesh. Left: $t=0.0$. Middle: $t=0.5$. Right: $t=1.0$.

$N_{0} \times M_{0}=20 \times 20$, the reaction rate (2.3) and the parameters $\gamma=1.2, q_{0}=50, E_{a}=50$, $K=2566.4$.

The colored contours in Fig. 4 show the density computed by the adaptive method and by nonadaptive methods. The adaptive method results are consistent with nonadaptive ones obtained with an $N_{0} \times M_{0}=160 \times 160$ uniform rectangular mesh. Fig. 5 shows the adaptive mesh at the time $t=0.2$. It is easily seen that the mesh refinement and coarsening follow the shock front. Moreover, compared to $P_{1}$-case, much more meshes got involved in the adaptive procedure in $P_{2}$-case. Note that $P_{1}$ and $P_{2}$ cases demonstrate similar features of the adaptive methods. Therefore, the case $P_{1}$ is omitted in what follows.

Example 4.3 (Stable ZND detonation - cf. Gao et al. [15]). Here, we model a stable ZND detonation up to the final time $t=30$ with the reaction rate (2.3) and the parameters $\gamma=1.1, q_{0}=2, E_{a}=20, K=1134363.64$. As a computational domain we use the rectangle $[130,230] \times[-5,5]$ and the detonation front is initially located at $x_{d}=225$. The initial condition in the $y$-component of velocity is perturbed by a transversely sinusoidal planar ZND wave 

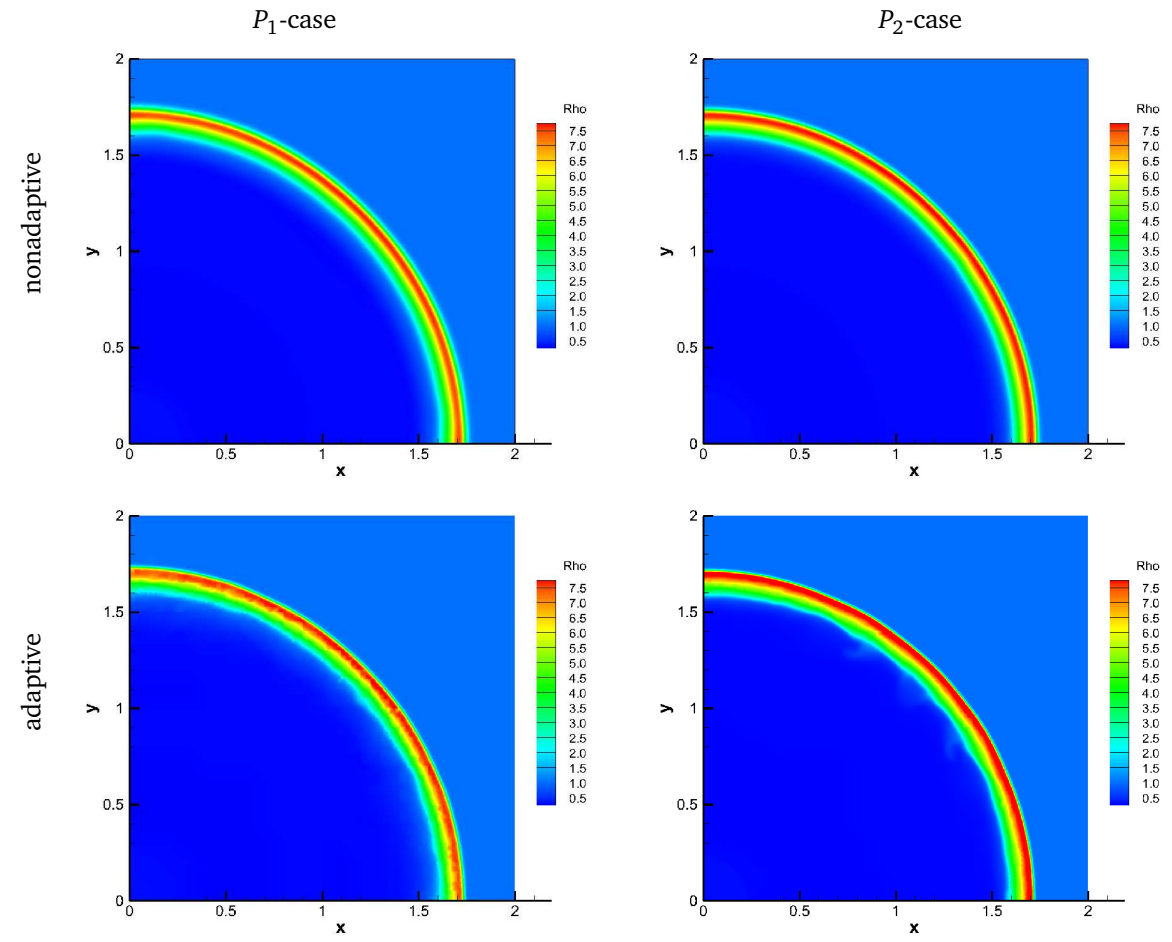

Figure 4: Example 4.2. Density contour, $t=0.2$. Top: Nonadaptive method. Bottom: Adaptive method. Left: $P_{1}$-case. Right: $P_{2}$-case.
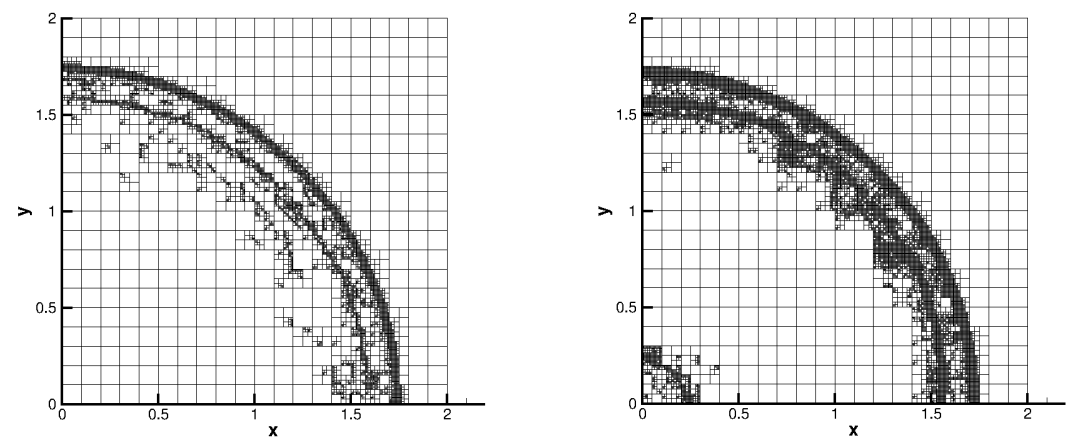

Figure 5: Example 4.2. Adaptive mesh, $t=0.2$. Left: $P_{1}$-case. Right: $P_{2}$-case.

density (adaptive)

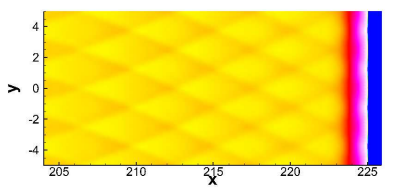

mesh (adaptive)

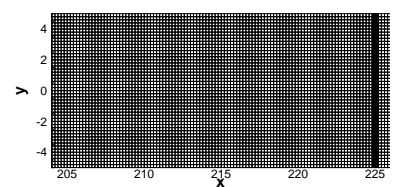

density (nonadaptive)

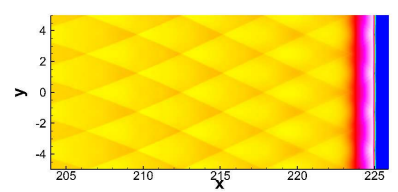

Figure 6: Example 4.3. $P_{2}$-case, $t=30$. Left: Density contours, adaptive method. Middle: Adaptive method mesh with a $500 \times 50$ initial mesh. Right: Density contours, nonadaptive method with $2000 \times 200$ mesh. 


$$
v(x, y, t=0)= \begin{cases}\frac{1}{10} \sin \left(\frac{4 \pi}{5} y\right), & 224 \leq x \leq 225, \\ 0, & \text { otherwise }\end{cases}
$$

The initial free stream and periodical boundary conditions are imposed in the $x$ - and $y$ directions respectively — cf. [14, 15, 37].

The colored contours in Fig. 6 display the density computed by the $P_{2}$ adaptive and non-adaptive methods. The initial $500 \times 50$ mesh in the adaptive method is shown in the left panel. The middle panel demonstrates the corresponding adaptive mesh. The nonadaptive method uses $2000 \times 200$ uniform mesh. Both methods established comparable regular cellular structures, but the resolution in the adaptive solution is slightly lower, since the corresponding mesh is refined along detonation front only.

Example 4.4 (Detonation diffraction problem - cf. Wang et al. [33]). The detonation diffraction phenomena is numerically challenging, especially for the high order schemes, mainly because the pressure or density may be very close to zero. To illustrate the good performance of the adaptive method, we consider a gaseous detonation waves passing through an obstacle with an $90^{\circ}$ angle turn. The initial conditions are

$$
\left(\rho, u, v, E, f_{1}\right)= \begin{cases}(11,6.18,0,970,1), & x<0.5, \\ (1,0,0,55,1), & \text { otherwise }\end{cases}
$$

and the final time is $t=0.6$. The boundary conditions are reflective, except the one at $x=0,\left(\rho, u, v, E, f_{1}\right)=(11,6.18,0,970,1)$. The parameters are $\gamma=1.2, q_{0}=50, E_{a}=50$, $K=2566.4$ with the reaction rate (2.3).

The colored contours in Fig. 7 display the density computed by the adaptive method initially based on $N_{0} \times M_{0}=50 \times 50$ uniform cells at the time instants $t=0.2,0.4,0.6$. The results are consistent with nonadaptive method using $N_{0} \times M_{0}=400 \times 400$ uniform cells and also with [33]. The corresponding temperature contours are presented in Fig. 8. They clearly expose the chemical reaction front. The corresponding adaptive mesh in Fig. 9 shows that the KXRCF troubled-cell indicator captures the shock location very well. The finest mesh along the shock generated by the adaptive method and the coarsest mesh used in the smooth region are also clearly visible.

Example 4.5 (Triple points problem — cf. Bao \& Jin [2]). The triple points travelling in the transverse direction and reflecting from the upper and lower walls are simulated cf. $[12,18,21]$. The initial conditions are chosen as

$$
\left(\rho, u, v, P, f_{1}\right)= \begin{cases}\left(\rho_{l}, 8.162 \cdot 10^{4}, 0, P_{l}, 0\right), & x \leq \xi(y), \\ \left(1.201 \cdot 10^{-3}, 0,0,8.321 \cdot 10^{5}, 1\right), & x>\xi(y),\end{cases}
$$

where

$$
\xi(y)= \begin{cases}0.004, & |y-0.0025| \geq 0.001 \\ 0.005-|y-0.0025|, & |y-0.0025|<0.001\end{cases}
$$



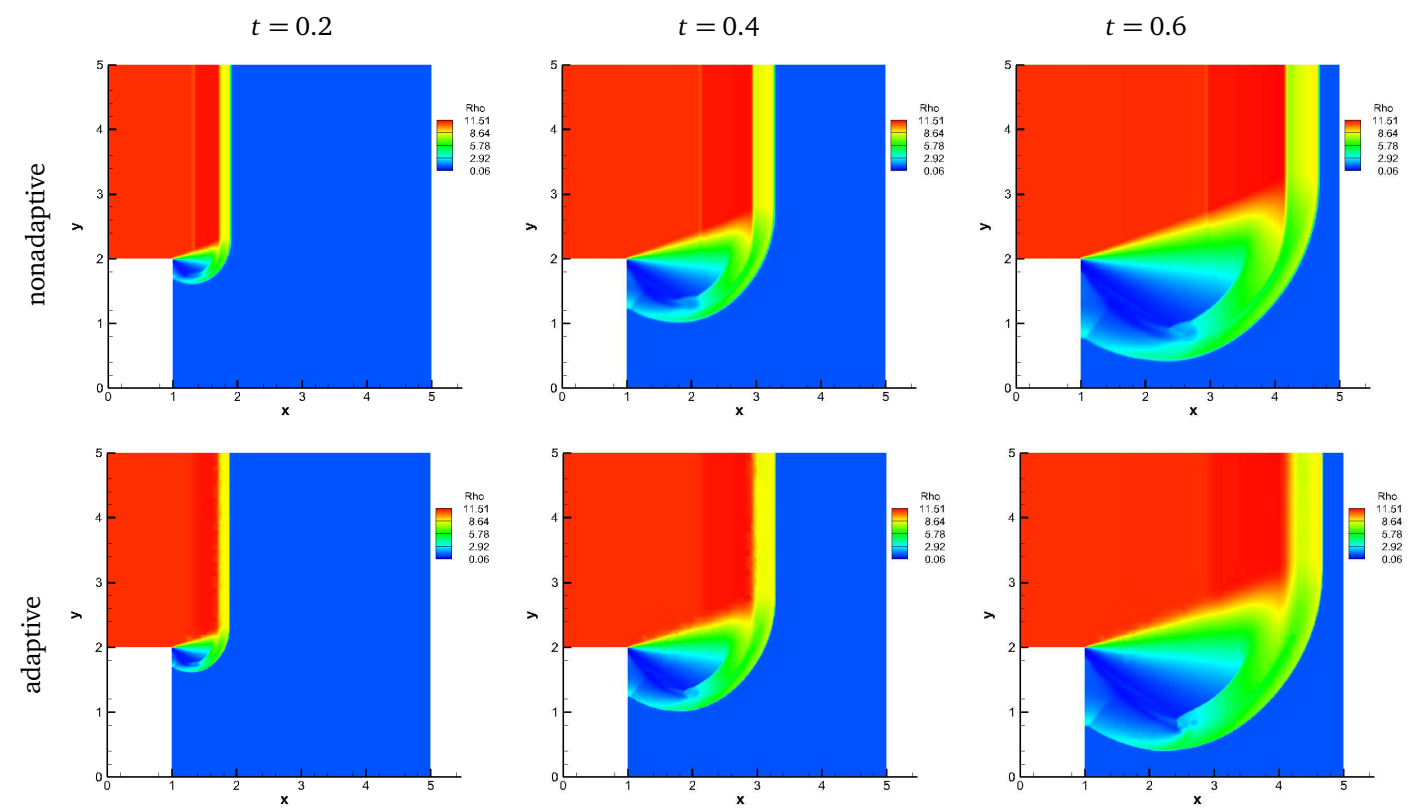

Figure 7: Example 4.4. Density contours at different time instants, $P_{2}$-case. Top: Nonadaptive method. Bottom: Adaptive method.

$t=0.2$

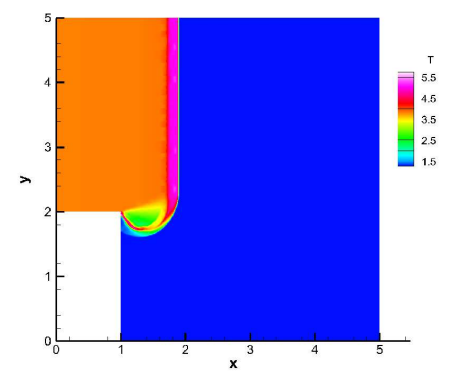

$t=0.4$

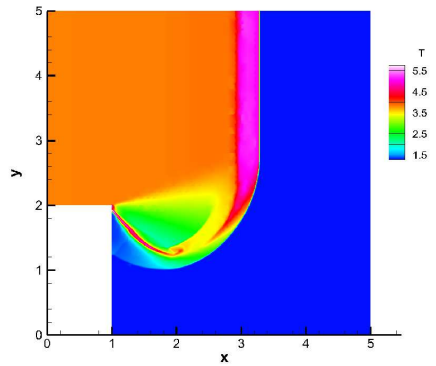

$t=0.6$

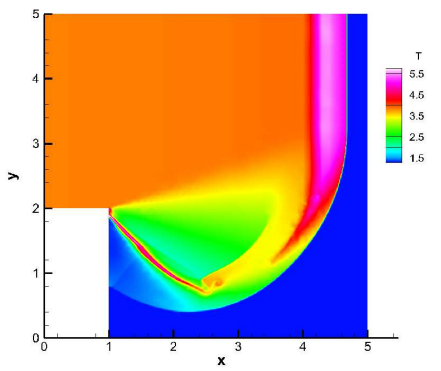

Figure 8: Example 4.4. Temperature contours, adaptive method for $P_{2}$-case at different time instants.
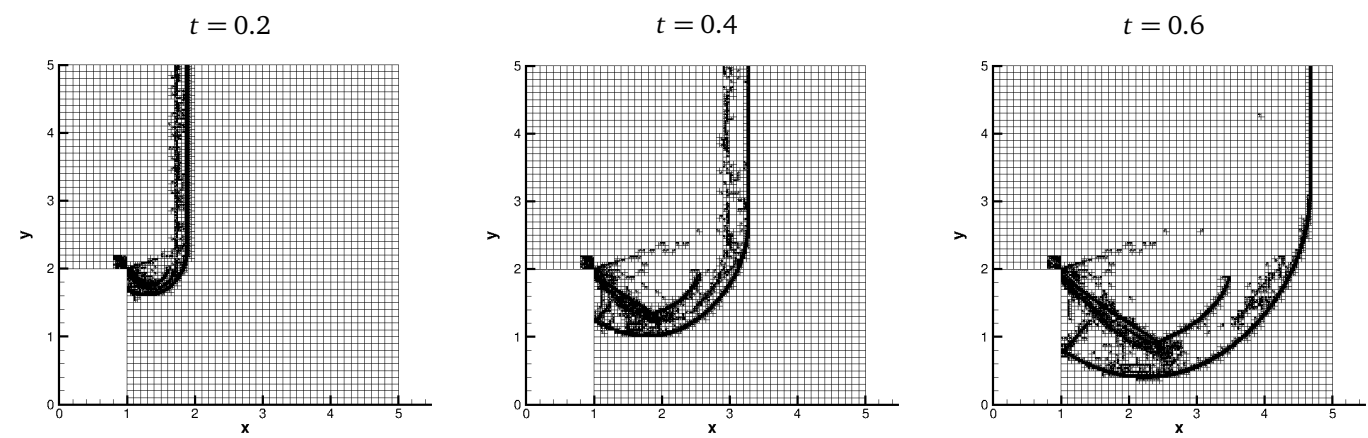

Figure 9: Example 4.4. Adaptive mesh temporal history, $P_{2}$-case. 
and $P_{l}, \rho_{l}$ have the form

$$
\begin{aligned}
& P_{l}=-b+\left(b^{2}-c\right)^{1 / 2}, \\
& \rho_{l}=\frac{\rho_{u}\left[P_{l}(\gamma+1)-P_{u}\right]}{\gamma P_{l}},
\end{aligned}
$$

where

$$
\begin{aligned}
& b=-P_{u}-\rho_{u} q_{0}(\gamma-1), \\
& c=P_{u}^{2}+2(\gamma-1) P_{u} \rho_{u} q_{0} /(\gamma+1) .
\end{aligned}
$$

Besides, we use the reaction rate (2.4) and the parameters $\gamma=1.4, q_{0}=0.5196 \times 10^{10}$, $1 / \sigma=0.5825 \times 10^{10}, E_{a}=0.1155 \times 10^{10}$.

The colored contours in Fig. 10 display the density computed by the adaptive and nonadaptive methods at the time instants $t=2 \times 10^{-8}, 4 \times 10^{-8}, 6 \times 10^{-8}, 8 \times 10^{-8}, 10 \times 10^{-8}$. The adaptive method based on the initial $N_{0} \times M_{0}=200 \times 40$ uniform cells is comparable with the nonadaptive one on $N_{0} \times M_{0}=1600 \times 320$ uniform grid. It is easily seen that the complex structures are generated by propagation of triple points and reflective waves from walls. The corresponding adaptive mesh temporal history is presented in Fig. 11. It shows the finest mesh used to resolve the complex structures.

To demonstrate other features of the adaptive method, the corresponding mesh data are presented in Fig. 12. We note that in the adaptive method, the number of cells increases with the time. It is consistent with the evolution of shock waves, which grows as time increases. Moreover, for comparable solutions in Examples 4.2, 4.3 and 4.4, the adaptive method outperforms the nonadaptive one in terms of computational storage. However, in Example 4.5 the number of cells in the adaptive method becomes larger than in nonadaptive ones. This happens because a complicated solution structure appears in the region where the detonation front passes through and a fully refined mesh shall be generated almost everywhere there - cf. Fig. 11. For $P_{2}$-case, much more grid points than for $P_{1}$-case got involved in the adaptive procedure, since the KXRCF indicator usually detects more troubled cells in $P_{2}$-case $[24,39]$.

\section{Concluding Remarks}

We developed an $h$-adaptive Runge-Kutta discontinuous Galerkin (RKDG) method with a positivity-preserving technique to simulate several classical two-dimensional detonation waves. The KXRCF troubled-cell indicator is used to detect the troubled cells with possible discontinuities or high gradients. At each time-level, an adaptive mesh is generated by refining troubled cells and coarsening others. In order to avoid the situations where detonation front moves too fast and there are not enough cells to describe detonation front before it leaves, a recursive multi-level mesh refinement technique is designed. The numerical results show that for smooth solutions this $h$-adaptive method does not degrade the optimal convergence order of the nonadaptive method and outperforms it in terms of computational storage for shocked flows. 

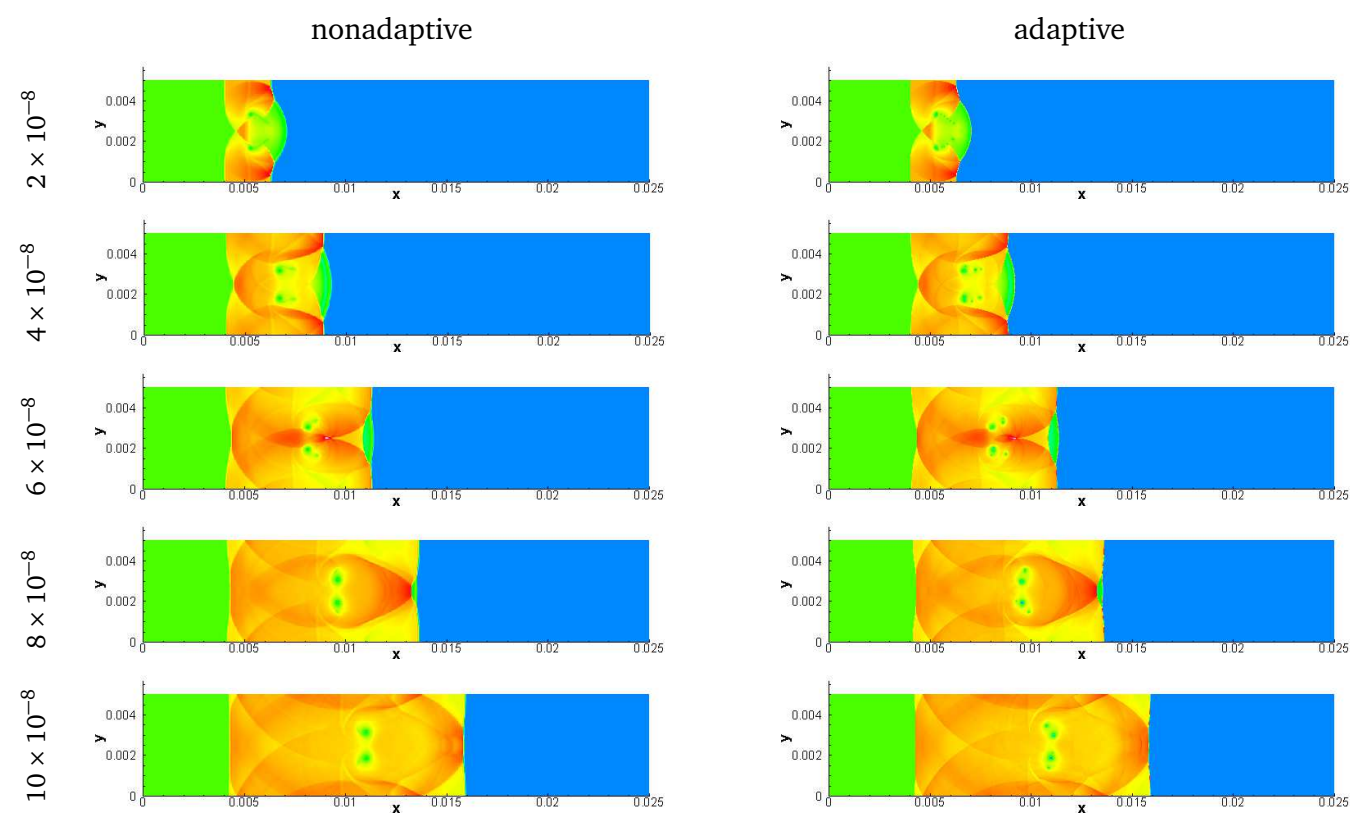

Figure 10: Example 4.5. Density contours at different time instants, $P_{2}$-case. Left: Nonadaptive method. Right: Adaptive method.

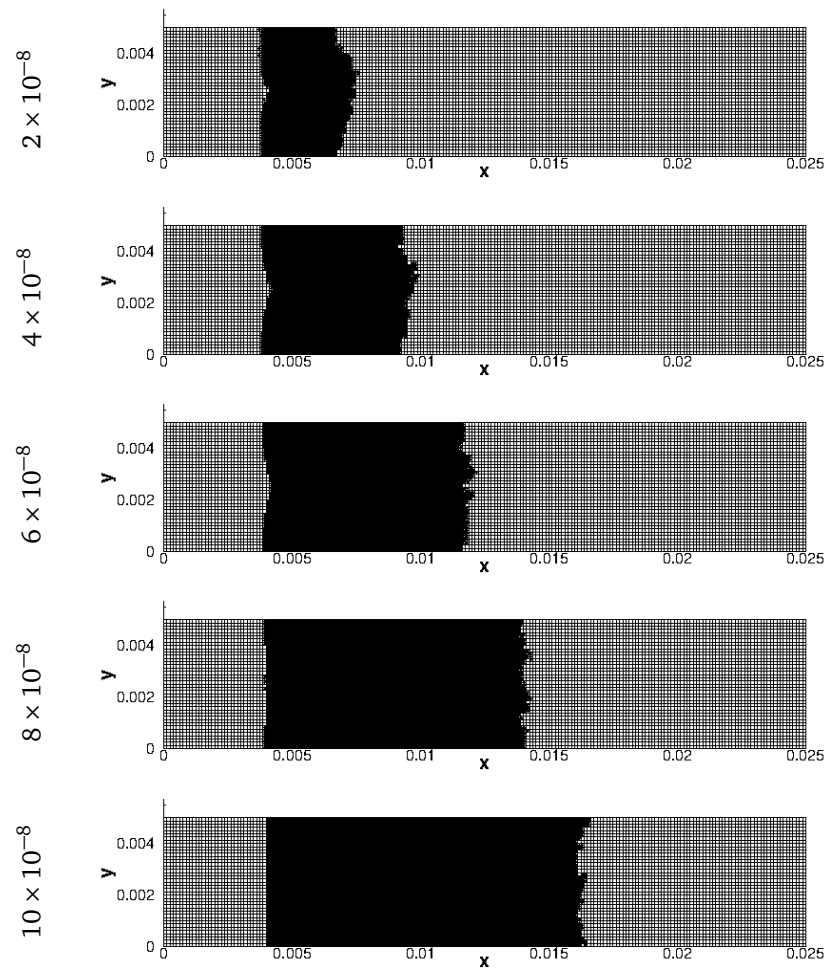

Figure 11: Example 4.5. Adaptive mesh history, $P_{2}$-case. 


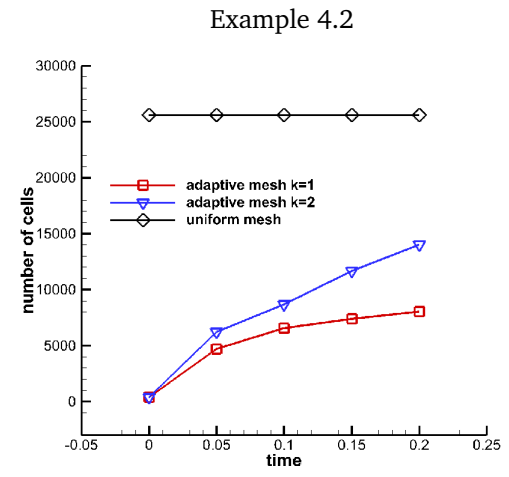

Example 4.4

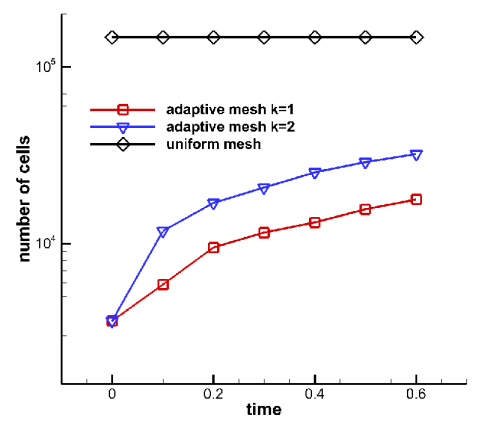

Example 4.3

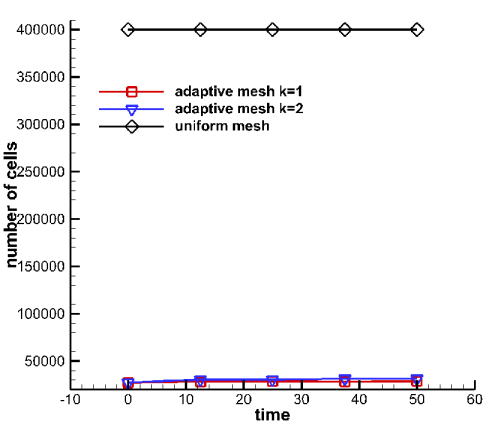

Example 4.5

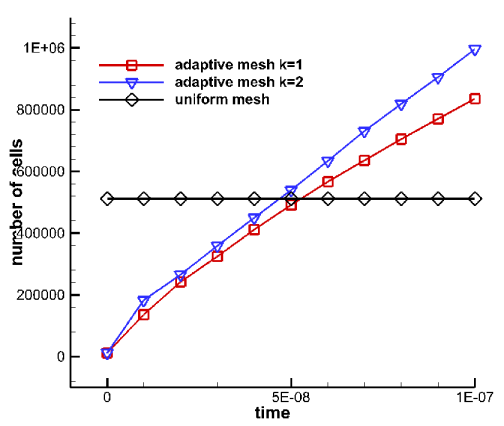

Figure 12: Number of cells at different time instants.

\section{Acknowledgments}

One of us (H.Z.) is partially supported by the Natural Science Foundation for Colleges and Universities in Jiangsu Province (17KJB110013), and the other (Z.G.) is partially supported by the National Science and Technology Major Project (20101010), the Shandong Provincial Natural Science Foundation (ZR2017MA016) and the Fundamental Research Funds for the Central Universities (201562012). Z.G. also thanks the Peking University for providing the funding support (Computational Analysis of Compressible Turbulence: 00110040). Both authors also acknowledge the funding support of this research by National Natural Science Foundation of China (11871443).

\section{References}

[1] B. Azarenok and T. Tang, Second-order Godunov-type scheme for reactive flow calculations on moving meshes, J. Comput. Phys. 206, 48-80 (2005).

[2] W. Bao and S. Jin, The random projection method for hyperbolic conservation laws with stiff reaction terms, J. Comput. Phys. 163, 216-248 (2000).

[3] G. Billet, J. Ryan and M. Borrel, A Runge Kutta Discontinuous Galerkin approach to solve reactive flows on conforming hybrid grids: the parabolic and source operators, Comp. Fluid. 95, 98-115 (2014). 
[4] B. Cockburn, S. Hou and C.-W. Shu, The Runge-Kutta local projection discontinuous Galerkin finite element method for conservation laws IV: The multidimensional case, Math. Comp. 54, 545-581 (1990).

[5] B. Cockburn, G. Karniadakis and C.-W. Shu, The development of discontinuous Galerkin methods, in: Discontinuous Galerkin Methods: Theory, Computation and Applications, B. Cockburn, G. Karniadakis and C.-W. Shu (Eds), pp. 3-50, Lecture Notes in Computational Science and Engineering 11, Springer (2000).

[6] B. Cockburn, S.-Y. Lin and C.-W. Shu, TVB Runge-Kutta local projection discontinuous Galerkin finite element method for conservation laws III: One dimensional systems, J. Comput. Phys. 84, 90-113 (1989).

[7] B. Cockburn and C.-W. Shu, TVB Runge-Kutta local projection discontinuous Galerkin finite element method for conservation laws II: General framework, Math. Comp. 52, 411-435 (1989).

[8] B. Cockburn and C.-W. Shu, The Runge-Kutta discontinuous Galerkin method for conservation laws V: Multidimensional systems, J. Comput. Phys. 141, 199-224 (1998).

[9] C. Dawson and R. Kirby, High resolution schemes for conservation laws with locally varying time steps, SIAM J. Sci. Comput. 22, 2256-2281 (2001).

[10] A. Dedner, C. Makridakis and M. Ohlberger, Error control for a class of Runge-Kutta discontinuous Galerkin methods for nonlinear conservation laws, SIAM J. Numer. Anal. 45, 514-538 (2007).

[11] H.S. Dou, H.M. Tsai, B.C. Khoo and J. Qiu, Simulations of detonation wave propagation in rectangular ducts using a three-dimensional WENO scheme, Combust. Flame 154, 644-659 (2008).

[12] B. Engquist and B. Sjogren, Robust difference approximations to stiff inviscid detonation waves, CAM Report 91-03, UCLA (March 1991).

[13] Z. Gao and W.S. Don, Mapped hybrid central-WENO finite difference scheme for detonation waves simulations J. Sci. Comput. 55, 351-371 (2013).

[14] Z. Gao, W.S. Don and Z. Li, High order weighted essentially non-oscillation schemes for onedimensional detonation wave simulations, J. Comput. Math. 29, 623-638 (2011).

[15] Z. Gao, W.S. Don and Z. Li, High order weighted essentially non-oscillation schemes for twodimensional detonation wave simulations, J. Sci. Comput. 53, 80-101 (2012).

[16] R. Hartmann and P. Houston, Adaptive discontinuous Galerkin finite element methods for nonlinear hyperbolic conservation laws, SIAM J. Sci. Comput. 24, 979-1004 (2002).

[17] G. Hu, A numerical study of $2 D$ detonation waves with adaptive finite volume methods on unstructured grids, J. Comput. Phys. 331, 297-311 (2017).

[18] K. Kailasanath, E.S. Oran, J.P. Boris and T.R. Young, Determination of detonation cell size and the role of transverse waves in two-dimensional detonations, Combust. Flame 61, 199-209 (1985).

[19] L. Krivodonova. An efficient local time-stepping scheme for solution of nonlinear conservation laws, J. Comput. Phys. 229, 8537-8551 (2010).

[20] L. Krivodonova, J. Xin, J.F. Remacle, N. Chevaugeon and J. Flaherty, Shock detection and limiting with discontinuous Galerkin methods for hyperbolic conservation laws, Appl. Numer. Math. 48, 323-338 (2004).

[21] C.B. Lee and J.Z. Wu, Transition in wall-bounded flows, Appl. Mech. Rev. 61, 030802 (2008).

[22] L. Liu, X. Li and F.Q. Hu, Nonuniform time-step Runge-Kutta discontinuous Galerkin method for computational aeroacoustics, J. Comput. Phys. 229, 6874-6897 (2010).

[23] M.V. Papalexandris, A. Leonard and P.E. Dimotakis, Unsplit schemes for hyperbolic conservation laws with source terms in one space dimension, J. Comput. Phys. 134, 31-61 (1997).

[24] J. Qiu and C.W. Shu, A comparison of troubled-cell indicators for Runge-Kutta discontinuous Galerkin mehtods using weighted essentially nonosillatory limiters, SIAM J. Sci. Comput. 27, 
995-1013 (2005).

[25] J. Qiu and C.W. Shu, Runge-Kutta discontinuous Galerkin method using WENO limiters, SIAM J. Sci. Comput. 26, 907-929 (2005).

[26] J.-F. Remacle, J. Flaherty and M. Shephard, An adaptive discontinuous Galerkin technique with an orthogonal basis applied to compressible flow problems, SIAM Review 45, 53-72 (2003).

[27] G.J. Sharpe and S.A.E.G. Fall, Two-dimensional numerical simulations of idealized detonations, Proc. R. Soc. 456, 2081-2100 (2000).

[28] C.W. Shu and S. Osher, Efficient implementation of essentially non-oscillatory shock-capturing schemes, J. Comput. Phys. 77, 439-471 (1988).

[29] L. Tian, Y. Xu, J.G.M. Kuerten and J.J.W. van der Vegt, An h-adaptive local discontinuous Galerkin method for the Navier-Stokes-Korteweg equations, J. Comput. Phys. 319, 242-265 (2016).

[30] E.F. Toro and V.A. Titarev, ADER schemes for scalar non-linear hyperbolic conservation laws with source terms in three-space dimensions, J. Comput. Phys. 202, 196-215 (2005).

[31] N. Tsuboi, Y. Daimon and A.K. Hayashi, Three-dimensional numerical simulation of detonations in coaxial tubes, Shock Waves 18, 379-392 (2008).

[32] C. Wang, J. Ning and T. Ma, Numerical simulation of detonation and multi-material interface tracking, Comput. Mater. Con. 22, 73-96 (2011).

[33] C. Wang, X. Zhang, C.W. Shu and J. Ning, Robust high order discontinuous Galerkin schemes for two-dimensional gaseous detonations, J. Comput. Phys. 231, 653-665 (2012).

[34] L. Yuan and L. Zhang, A Runge-Kutta discontinuous Galerkin method for detonation wave simulation, AIP Conf. Proc. 376, 543-545 (2011).

[35] Z.C. Zhang, S.T. Yu, H. He and S.C. Chang, Direct calculation of two- and three- dimensional detonations by an extended CE/SE method, AIAA, 2001-0476 (2001).

[36] J. Zhao and H. Tang, Runge-Kutta central discontinuous Galerkin methods for the special relativisitic hydrodynamics, Commun.Comput. Phys. 22, 643-682 (2017).

[37] H. Zhu and Z. Gao, An h-adaptive RKDG method with troubled-cell indicator for one-dimensional detonation wave simulations, Adv. Comput. Math. 42, 1081-1102 (2016).

[38] H. Zhu and J. Qiu, Adaptive Runge-Kutta discontinuous Galerkin methods using different indicators: One-dimensional case, J. Comput. Phys. 228, 6957-6976 (2009).

[39] H. Zhu and J. Qiu, An h-adaptive RKDG method with troubled-cell indicator for two-dimensional hyperbolic conservation laws, Adv. Comput. Math. 39, 445-463 (2013).

[40] H. Zhu and J. Qiu, An h-adaptive Runge-Kutta discontinuous Galerkin method for HamiltonJacobi equations, Numer. Math. Theor. Meth. Appl. 6, 617-636 (2013).

[41] J. Zhu, X. Zhong, C.W. Shu and J. Qiu, Runge-Kutta discontinuous Galerkin method with a simple and compact Hermite WENO limiter on unstructured meshes, Commun.Comput. Phys. 21, 623-649 (2017). 